\title{
DISKUSSION
}

\section{Aus Anlaß einer Rezension}

Juhani Lehtiranta hat in Band XLVII der Finnisch-Ugrischen Forschungen meine Dissertation über den ältesten finnischen Wortschatz und dessen Erforschung rezensiert. Da ich das Buch bereits vor langer Zeit geschrieben habe, möchte ich nicht mehr auf dessen Einzelheiten eingehen. Ich fordere jedoch die Leser der Rezension auf, die kommentierten Stellen auch im Original zu überprüfen, da bei Lehtiranta die angeführten Stellen vielfach mangelhaft referiert oder interpretiert oder aber Details unnötig aufgebauscht werden. Hierfür nenne ich einige Beispiele, die meine Aufforderung unterstützen können.

Auf die sprachwissenschaftlichen Grundlagen der Stammbaumtheorie oder besser auf das Fehlen solcher Grundlagen gehe ich hier nicht ein, da ich mich mit dem Thema in einem umfangreichen Aufsatz nach dem Erscheinen meiner Dissertation auseinandergesetzt habe (UAJb 4, Neue Folge, 1984). Lehtiranta hat meines Erachtens keine neuen stichhaltigen Argumente für die Stammbaumhypothese vorgebracht. Die Glottochronologie, wie Lehtiranta sie derzeit in seinen eigenen Untersuchungen anwendet, ist als Methode bedenklich, da ihre Zuverlässigkeit bei jeder Anwendung mittels anderer Methoden abzuschätzen ist. Eine taugliche Methode müßte jedesmal zuverlässige Resultate ergeben. Wie ich in meiner Dissertation erwähne, hat man besonders in der Romanistik die unbestreitbare Unvereinbarkeit von historischer Forschung und glottochronologischer Methode nachweisen können. Was soll nun in Frage gestellt werden, die historischen Dokumente oder die Glottochronologie? Ist die Methode entschieden besser bei der Erforschung vorhistorischer Entwicklungsphasen oder sieht sie nur besser aus, da man sie nicht testen kann? Der mit der Glottochronologie vertraute Leser weiß, daß von den Wörterlisten des Morris Swadesh zahlreiche, im Umfang etwas voneinander abweichende Versionen verwendet werden und daß die umfangreichsten aus Gründen der Bequemlichkeit als 200-Wörter-Listen bezeichnet werden, auch wenn die reale Anzahl der Wörter nicht unbedingt genau 200 beträgt.

Den Terminus "pensasmalli (Strauch-, Buschmodell)" habe ich lediglich in Anführungszeichen verwendet, wenngleich ich bereits ahnte, daß ein Leser, der für seine Gedanken konkrete Fixpunkte braucht, nur zu gern danach greifen würde und darin eine Entsprechung für den Stammbaum und die Darstellung von der Auflösung der uralischen Ursprache sehen würde. Graphisch erinnert der von mir dargestellte "Strauch" zunächst an einen Kamm: in Aufbau und Zweck entspricht er in erster Linie jenen graphischen Darstellungen, mit deren Hilfe man auch die Zusammensetzung der indogermanischen Sprachgemeinschaft zu visualisieren pflegt. Das Modell soll zunächst betonen, daß wir über den vermeintlichen Auflösungsprozeß der hypothetischen uralischen Ursprache nichts wissen, was so sicher wäre, daß es den Ausgangspunkt für weitreichende Schlußfolgerungen abgeben könnte. Der entscheidende Vorteil des "Strauchmodells" verglichen mit dem Stammbaummodell liegt jedenfalls darin, daß der gemeinsame Stamm bzw. die aufeinander folgenden $Z$ wischen-Ursprachen fehlen. Wenn man den Strauch 
untersucht, muß man nicht unbedingt auch einen jeden Zweig für sich untersuchen; kein $Z$ weig braucht eine Sonderstellung zu erhalten. Auch die Indogermanisten untersuchen in der Praxis ja nicht jede einzelne existierende indogermanische Sprache! Ich bin übrigens der Ansicht, daß wir in Zukunft die sprachgeschichtlich relevantesten Aufschlüsse über das Finnische erhalten, wenn wir uns die Entwicklung der finnischen Sprache in der Sprachgemeinschaft des Ostseeraums ansehen. Die Forschung müßte dort einsetzen, wo mit größter Wahrscheinlichkeit die sichersten Informationen zu holen sind, anstatt im Dunkel der Vorgeschichte zu suchen. Wenn man eine Hypothese auf die andere baut, kumuliert nur die Unsicherheit. Stellen wir uns eine Rechenaufgabe vor, von der nur das Endergebnis bekannt ist! Falls man nichts darüber weiß, wieviele und welche Rechenoperationen durchgeführt wurden und unter Verwendung welcher Zahlen, gibt es unzählige Möglichkeiten, die Aufgabe "richtig" zu lösen. Wenn erst einmal Zahlen und Rechenoperationen eingesetzt werden, beginnen die Entscheidungen einander natürlich zu beeinflussen, doch ändert das nichts an der Grundkonstellation. Alle uralischen Sprachen sind chronologisch gleich weit von ihrer gemeinsamen Ursprache entfernt, falls sie überhaupt eine haben, und es ist alles andere als klar, daß sich die Lösungen für die Rekonstruktionsprobleme der uralischen Ursprache ausdrücklich in den samojedischen Sprachen finden ließen. Der einzige wirklich gewichtige Grund, weshalb speziell ein Vergleich zwischen Ostseefinnisch und Samojedisch als exzeptionell wichtig anzusehen ist, liegt in ihrer geographischen Entfernung voneinander und dem offensichtlichen Mangel an Kontakten. Bei der Rekonstruktion des ururalischen Wortschatzes und Vokalismus meint Juha Janhunen (JSFOu 77) die ugrischen Sprachen völlig außer acht lassen zu können. Wie kann man wissen, ob die Unterschiede zwischen den hypothetischen Sprachformen, dem Ursamojedischen und der finnisch-permischen Ursprache, gerade aufs Ururalische zurückgehen oder ob sie nicht möglicherweise Innovationen eines der beiden Zweige darstellen? Und wenn dem so ist, woher weiß man, welches $Z$ weiges? Und besteht nicht die Möglichkeit, daß die Innovationen beiderseits die gleichen Eigenschaften vorgefunden haben? Hinsichtlich der Berechtigung seines Verfahrens beruft Janhunen sich auf die allgemein bekannte Konservativität an der äußersten Peripherie der Sprachgemeinschaft. Könnte man nicht ebenso gut behaupten, daß man am äußersten Rand der Sprachgemeinschaft höchstwahrscheinlich mit Vertretern anderer Sprachgemeinschaften Kontakt gehabt hat? Trifft das nicht beispielsweise gerade für die Sprecher der ostseefinnischen Sprachen zu? Lehtiranta beruft sich eifrig auf Janhunen, wann immer es zu passen scheint; die weniger passenden Stellen läßt er unberücksichtigt. Lehtiranta selbst betont den innovativen Charakter des Ostseefinnischen. Daß das Ostseefinnische in der Erforschung der Vokalgeschichte die Schlüsselsprache bildet, beruht andererseits gerade auf der besonderen Konservativität des Finnischen (s. z. B. E. Itkonen UAJb 41, 76 - 111). Janhunen erwähnt in seinem Aufsatz nicht, wem die feststehenden Ansichten über den Vokalismus der finnisch-permischen Ursprache zu verdanken sind, aber zweifellos meint er Erkki Itkonen. Jedenfalls stellt Janhunen fest, eine Rekonstruierung des Ururalischen sei erst jetzt sinnvoll, nachdem die Vokalgeschichte der finnisch-permischen Sprachen in ihren Hauptzügen geklärt worden sei. In seiner eigenen Untersuchung vergleicht er nicht mehr die finnisch-permischen Sprachen mit den samojedischen Sprachen, sondern den rekonstruierten Vokalismus der finnisch-permischen Ursprache mit dem rekonstru- 
ierten Vokalismus des Ursamojedischen. Was aber geschieht, wenn sich die Meinungen über den konservativen Charakter des Finnischen und über seine Position als Schlüsselsprache ändern? Und wenn die Einbeziehung der ugrischen Sprachen in den Vergleich zu einer Änderung der Auffassung über die Entwicklung auch der anderen Sprachen führt?

Die verschiedenen Quellen geben unterschiedliche Angaben. In zahlreichen Fällen, wo Lehtiranta die Wahl gehabt hätte, scheint er ohne weiteres den sowjetischen Forschern den Vorrang zu geben. Weshalb weist er bei der Behandlung z. B. des *ne-Konjunktivs nicht darauf hin, daß u. a. Björn Collinder, Mikko Korhonen und vor allem Péter Hajdú über dessen Alter anderer Meinung sind als Majtinskaja und Janhunen? Zu Janhunens Darstellung über die Struktur der uralischen Ursprache (FUF 44) läßt sich schwer Stellung nehmen, da sie an vielen Stellen recht oberflächlich und undifferenziert gehalten ist. Janhunen bringt überhaupt nicht zur Sprache, daß der *ne-Konjunktiv von vielen Forschern als ururalisch angenommen wird; somit widerlegt er auch nicht jene Argumente, mit denen der Konjunktiv für das Ururalische rekonstruiert wurde. Er verweist auch nicht auf Majtinskaja, obleich im Literaturverzeichnis zwei Arbeiten von Majtinskaja über die finnisch-ugrischen [!] Sprachen erwähnt sind. An sich enthält der Aufsatz von Janhunen keinen einzigen Hinweis, was in wissenschaftlichen Darstellungen ziemlich ungewöhnlich ist.

Die Suffixe ${ }^{*} A$ und $* j A$ sind allgemein bekannte Beispiele für uralische deverbale Nominalsuffixe; wie der aufmerksame Leser merken kann, habe ich auch gar nicht versucht, sie als jünger hinzustellen. Es geht nur darum, wann sie zu einem Teil der finiten Flexion geworden sind. In dem von Lehtiranta angeführten Janhunen-Artikel werden die Suffixe ausdrücklich bei den nichtfiniten Formen behandelt, wenn auch allgemein festgestellt wird, daß zahlreiche derartige Elemente auch als Basis für die finite Flexion gedient haben.

Lehtiranta wundert sich, wieso ich den Stammbaum kritisiere und doch selbst auf S. 83 kommentarlos [!] ein Stammbaumdiagramm bringe. Die Kommentare finden sich auf der nebenstehenden Seite, desgleichen der Hinweis auf die Abbildung. Die Bildlegende gibt ferner die Quellen an, auf denen die Darstellung beruht.

Bei der Erörterung dessen, wie sinnvoll die nostratische Hypothese ist, stelle ich fest, daß eine in ihrem Entwicklungsniveau urtümliche Kultur kaum als große Sozietät fungieren kann, und daß eine Sozietät mit großer geographischer Verbreitung in diesen Verhältnissen kaum ihre sprachliche Einheitlichkeit beibehalten würde. Hierauf erwidert Lehtiranta, keine Kultur sei primitiv, da alle Kulturen das Ergebnis jahrtausendelanger Entwicklung seien. Die übrigen Leser meines Buches dürften verstanden haben, daß der von mir verwendete Ausdruck alkeellinen 'urtümlich, einfach' nicht auf die Dauer der Existenz hinweist, sondern auf den Grad der Entwickeltheit einer Kultur. In diesem Zusammenhang bedeutet das u. a., daß es keine raschen und mühelosen Mittel zur Aufnahme und Unterhaltung von Kontakten gibt, wie es in entwickelten Gemeinschaften z. B. die mit Rädern versehenen Transportmittel sind, die Transportwege, die Massenmedien usw. An dieser Stelle dürfte die Erwähnung angebracht sein, daß der von Lehtiranta häufig zitierte Janhunen am 6.4.1983 an der Universität Turku eine Vorlesung im Rahmen der Weiterbildung hielt, und zwar über die Probleme der genetischen und arealen Klassifizierung der Sprachen der Welt. In dieser Vorlesung stellte Janhunen $u$. a. fest, es gebe in Eurasien 
gerade deshalb beachtlich umfangreiche Sprachgemeinschaften, da die starken Kerngebiete der kulturellen Entwicklung eben in dieser Richtung zu finden sind. Die kulturellen Innovationen der neolithischen Steinzeit ermöglichten eine effiziente Zunahme der Bevölkerung und ihre Verbreitung in ausgedehnten Gebieten. Dasselbe sagte Janhunen auch, indem er feststellte, $\mathrm{da} ß$ ein umfangreiches zusammenhängendes Sprachgebiet als Zeichen einer relativ späten Expansion gedeutet werden kann. Ahnliche Gedanken hat auch Mikko Korhonen geäußert u. a. in dem Zusammenhang, der in der Anmerkung in meiner Dissertation steht, sowie später u. a. in seinem Aufsatz, der in der Publikation über die vorhistorischen Wurzeln der Population Finnlands (Suomen väestön esihistorialliset juuret. Bidrag till kännedom av Finlands natur och folk. H. 131. 1984) enthalten ist. Auf entsprechende Ideen kommt ohne weiteres auch jeder Dialektforscher, wenn er sich anschickt, die Gründe für die Verschiedenheiten der Dialekte zu erklären. Ich wage weiterhin zu behaupten, daß Sprachgemeinschaften mit einem niedrigen Entwicklungsniveau wahrscheinlich weder eine große räumliche Ausdehnung haben noch einheitlich sind. Wenn man sich auf die Suche nach den gemeinsamen Wurzeln der Bevölkerung und der Sprache Eurasiens begibt, handelt es sich nicht mehr um Expansionen der neolithischen Steinzeit, sondern um etwas, was meines Erachtens von der herkömmlichen historisch-vergleichenden Sprachforschung nicht mehr geleistet werden kann.

Nach Lehtiranta halte ich die Existenz einer ugrischen Ursprache für wahrscheinlich. An sich sage ich, daß die Wortschatzverhältnisse eher dafür als dagegen sprechen (S. 95). Nun kann man hier aber nicht willkürlich mit dem Lesen aufhören, denn die Behandlung der Frage geht bis zum Ende des Kapitels weiter. Ich stelle fest, daß die lexikalischen Beziehungen zwar von einer Verwandtschaft zeugen, über den Grad dieser Verwandtschaft aber nicht viel aussagen. Auf Ubereinstimmungen im Wortschatz läßt sich also die Verwandtschaftshypothese bauen, nicht aber die Stammbaumhypothese, und - wie man seit den Tagen von Sajnovics und Gyarmathi weiß - auch bei der Einschätzung der verwandtschaftlichen Beziehungen entscheiden die morphologischen Beziehungen und nicht die Anzahl der gemeinsamen Wörter. Aufgrund meiner eigenen lexikalischen Untersuchungen behaupte ich also überhaupt nichts über die Existenz des Urugrischen; ich stelle lediglich fest, daß die lexikalischen Gegebenheiten nicht ausreichen, um die von László Honti detailliert motivierten Auffassungen über die historische Wirklichkeit des Urugrischen zu widerlegen (ALH 29, NyK 81). Die Lückenhaftigkeit der Etymologien beweist, daß sich die ursprünglichen Verbreitungsverhältnisse nicht jahrtausendelang unverändert erhalten haben. Die Wörter haben sowohl am Rand der Verbreitungsgebiete als auch in den Zentren verschwinden können.

Lehtiranta stellt die Heterogenität des Finnischen Etymologischen Wörterbuches fest, als wäre dies etwas Neues. Lohnte sich diese Bemerkung, wo ich doch selbst mit Worten wie auch mit Abbildungen in meiner Dissertation darüber schreibe? Zumindest hätte auf diesen Tatbestand hingewiesen werden sollen, damit der Leser der Rezension nicht das Bild erhält, es handle sich hier um etwas Neues, noch nicht Festgestelltes.

Das Wort sata 'hundert' ist nach Lehtiranta kein Beweis für das Dezimalsystem. Was sollte es sonst sein, wenn es noch - nach Lehtiranta als $n^{2}$ definiert wird? Wenn das Wort in all den Sprachen, wo es realiter begegnet, dieselbe Bedeutung hat, besteht dann irgendein Anlaß, eine 
andere ursprüngliche Bedeutung anzunehmen? Etwas anderes ist natürlich, wann das Wort in Gebrauch kam. Ich nenne das Wort finnisch-ugrisch, weil es in allen finnisch-ugrischen Sprachen auftritt. Die Tatsache, daß dieselbe Zahl auch andere Benennungen hat, weist nicht auf einen Bedeutungswandel hin. Schießlich wurde ja auch in der alten finnischen Schriftsprache neben dem Wort miljoona 'Million' der Ausdruck tuhannen (kertaa) tuhatta 'tausend (mal) tausend' verwendet. Ich verstehe nicht, wieso das als Entsprechung für fiu. viisi ' 5 ' angeführte ursam. * wüt ' 10 ' als solches die Idee unterstützen soll, daß das ururalische Zahlensystem von der 5 ausgegangen sei, zumal es Hinweise auf größere Zahlenausdrücke gibt. Lehtiranta beruft sich wieder auf Janhunen; dieser hat für das ururalische Numerale sowohl die Bedeutung 'fünf' als auch 'zehn' rekonstruiert und erklärend hinzugefügt, das Wort habe den letzten Finger [also von der einen Hand oder von zwei Händen] beim Zählen bezeichnet. Von der Bedeutung 'fünf' gelangt man natürlich geschickt zu 'zehn', wenn man annimmt, daß das Wort im Samojedischen an sich der Dual des Wortes für 'fünf' ist. Ob das lautgeschichtlich unmöglich ist? Die Etymologie gilt auf jeden Fall schon jetzt als in vieler Hinsicht unregelmäßig, was die Lautverhältnisse betrifft. Da die Menschen zu allen Zeiten zehn Finger besaßen und da man zu allen Zeiten die Finger als Hilfsmittel beim Rechnen benutzt hat, ist 'zehn' meines Erachtens als Schlußpunkt beim Zählen natürlicher als 'fünf'. Dadurch wird nicht vorausgesetzt, daß ein regelrechtes Zehnersystem mit entsprechender Multiplikation in Gebrauch gewesen wäre, oder daß alle einmal verwendeten Zahlenbezeichnungen erhalten geblieben wären. Auch die Kultursprachen (u. a. Französisch) liefern Beispiele dafür, daß das Numeralsystem gründlich erneuert werden kann, obwohl die Zahlenbegriffe an sich beibehalten werden.

Den etymologischen Wortschatz wollte ich in meinem Buch aufnehmen, da ich selbst dessen Brauchbarkeit festgestellt hatte und hoffte, daß auch die Leser einen Nutzen davon hätten. Man kann leichter in einem kurzen, komprimierten Register nachschlagen als die Wortartikel von drei etymologischen Lexika durchgehen, die nach verschiedenen Prinzipien geschrieben sind. Ich habe nicht daran gedacht, daß dieser Wortschatz bereits existierende oder künftige Wörterbücher ersetzen sollte. Der Fachlexikologe verlangt natürlich nach umfassenderen Darstellungen, doch reicht in vielen Fällen auch die Information, in welchen Sprachen das betreffende Wort etymologische Entsprechungen hat und als wie sicher diese Etymologien in den wichtigsten etymologischen Wörterbüchern bezeichnet werden. Oft begnügt man sich ja mit viel weniger, wenn man z. B. auf ein einziges Wörterbuch, eine einzige Untersuchung hinweist. Vielleicht fühlt sich Lehtiranta durch meine konkrete Darstellungsweise irritiert, doch wird der Wert einer Untersuchung meines Erachtens nicht unbedingt durch die Schwererklärbarkeit erhöht. Was nützen abstrakte Gedankengebäude, die außer dem Verfasser niemand versteht und anwenden kann?

Lehtiranta wirft mir vor, daß ich für die finnisch-ugrischen Sprachen nicht die Eigenbenennungen verwende. Warum sollte ich? Das Selbstgefüh! vieler kleinen Minderheitsvölker mag dadurch steigen, daß ihre eigensprachlichen Benennungen auch in der herrschenden Sprache ihres großen Heimatlandes verwendet werden. Aber wenn das z. B. in der Sowjetunion geschieht, ist das wohl noch kein zwingender Grund, auch die Praxis in Finnland zu ändern. Schließlich werden im Russischen ja auch nicht die Eigenbennungen der Finnen und Ungarn für diese Völker und Sprachen verwendet, geschweige 
denn in den sonstigen Kultursprachen von Europa. Und wenn man damit begänne, würde das kaum zur Hebung des Selbstgefühls beitragen. Eher würde ein Bild von irgendwelchen merkwürdigen entlegenen Gegenden entstehen, für die es in den Kultursprachen noch nicht einmal einen Namen gibt. Ich verwende die Benennungen, an die ich gewöhnt bin, die im überwiegenden Teil der von mir benutzten Literatur verwendet werden und die derzeit auch in der finnischsprachigen Literatur frequenter sind als die Eigenbenennungen. Vom letztgenannten Tatbestand kann man sich überzeugen, wenn man z. B. "Maailman kielet" (Die Sprachen der Welt) von Aulis J. Joki anschaut, Mikko Korhonens hervorragende "Johdatus lapin kielen historiaan" (Einführung in die Geschichte des Lappischen), die nach dem interdisziplinären Symposion von Tvärminne erschienene Publikation "Suomen väestön esihistorialliset juuret" (Die vorgeschichtlichen Wurzeln der Bevölkerung Finnlands) oder die brandneue Enzyklopädie Facta 2001. [Welche Namen die Uralisten wählen, die in einem kleinen Kreis füreinander schreiben, hat keine große Bedeutung, zumal es normalerweise auf englisch oder deutsch geschieht.]

Viele der Uberschriften in Lehtirantas Rezension passen stilistisch eher in eine Jugendzeitschrift als in ein angesehenes wissenschaftliches Periodikum. Sachtext setzt sachlichen Stil voraus. Als Antwort hier ein Zitat aus der Vorrede zum Gebetbuch von Agricola:

MWtamat ylenwisut ouat. muijdhen töijsse Domarit couat Ja quin Sokia eroijtta caruat nijn tyhmet wisust kiriat aruat. Yxi sano ombi wäret. toijnen tas laijtta ilman märet. Colmas hylke, ia caiki sylke Eijkö caike, on alghus heicko? Nijn taij mös on, o hyue weicko Jos Jotakin on sijrtty toijsin. wehetty, lisetty, taicka poijsi. Eij taijdha caike olla teusi. sijs nautitze se iongas leusi. Tee parambi ios sine taijdhat. et te nämet ouat ylen caijdhat Ele polghe Kiria quin Sica. waicka henes on wehe wica.

Manche sind übergenau, strenge Kritiker der Arbeiten anderer. Und wie der Blinde die Farben unterscheidet, so genau verstehen die Dummen die Bücher.

Der eine sagt: Das ist falsch, der andere wiederum tadelt nach Belieben. Der dritte lehnt ab und spuckt auf alles. Ist nicht alles am Anfang schwach?

So auch dies hier, lieber Freund.

Wenn etwas verändert ist, vermindert, hinzugefügt oder fehlt. 